Proceedings of the 31st Annual Meeting of the Brazilian Embryo Technology Society (SBTE); Cabo de Santo Agostinho, PE, Brazil, August 17th to 19th, 2017.

\title{
Brazilian embryo industry in context: pitfalls, lessons, and expectations for the future
}

\author{
João Henrique Moreira Viana ${ }^{1,4}$, Ana Cristina Silva Figueiredo ${ }^{2}$, Luiz Gustavo Bruno Siqueira ${ }^{3}$ \\ ${ }^{1}$ Embrapa Recursos Genéticos e Biotecnologia, Brasília, DF, Brazil. \\ ${ }^{2}$ Universidade Jose do Rosario Vellano, Alfenas, MG, Brazil. \\ ${ }^{3}$ Embrapa Gado de Leite, Juiz de Fora, MG, Brazil.
}

\begin{abstract}
The aim of this review is to provide a brief description of the current situation of the embryo industry in Brazil, in the context of the national and international livestock production scenario. Total number of embryos produced $(375,894)$ declined in 2015 in comparison with the previous years. B. taurus dairy breeds and crossbreds accounted for $51.8 \%$ of the total embryo production. Moreover, the percentage of frozen-tawed embryos transferred reached $22.8 \%$ in 2015 , the highest value in a decade. A greater proportion of embryos were produced in vitro, both in dairy $(97.2 \%)$ and beef $(90.2 \%)$ breeds. The use of embryo technologies in Brazil has remarkably increased $726.5 \%$ in the past 20 years, but still represents only $0.33 \%$ of the number of cows and heifers at reproductive age. Nonetheless, embryo transfer (ET) accounted for an estimated $19.7 \%$ of all purebred calves born and registered by the Brazilian Zebu Cattle Breeders Association in the period 2005-2015, highlighting the importance of ET for animal breeding and genetic improvement of the herd. In the world's context, Brazil is the largest producer of bovine in vitro produced (IVP) embryos, but is ranked only 11th based on an index of intensity of use of embryo technologies, below Canada, USA and various European countries. This scenario demonstrates a potential for further increases in embryo production in Brazil, mainly associated with the expected adoption of new technologies by a large proportion of dairy and beef farms; the use of embryo technologies for large scale production of crossbreds; and to eventual increases in the international embryo import/export activity.
\end{abstract}

Keywords: cattle production, embryo technologies, statistics.

\section{Introduction}

The Brazilian embryo industry underwent remarkable changes in the past 15 years, most of them associated to the adoption of in vitro technologies. In cattle, there was a $>5$-fold increase in embryo production, and in vitro fertilization/culture has almost fully replaced superovulation (MOET) as the technique of choice for embryo production. Development of IVP in Brazil was addressed in previous studies (Viana et al., 2010, 2012; Sartori et al., 2016), with a main focus on the technical aspects of the technology. The use of embryo transfer, however, affects overall cattle production both by increasing genetic progress of animal breeding programs and by providing new alternative methods to produce crossbred animals.

Nevertheless, changes in dairy and beef production systems, induced by the pressure to increase productivity, reduce costs, or improve animal welfare will probably drive further demands for the development of embryo technologies. Thus, to understand the past contribution and the prospective expectations of Brazilian embryo industry, records and numbers must be analyzed in a context. In this study we present a brief description of the current situation of embryo production in Brazil, as well as some proposed indexes to characterize activities related to embryo technologies in the national and international scenario.

\section{A summary of changes in the Brazilian embryo industry during the past decade}

Data of Brazilian embryo production in 2015, discriminated by genetic group (Bos taurus and Bos indicus), industry (dairy and beef) and technology used (in vivo derived - IVD or in vitro produced - IVP) is shown in Table 1. A decline in the number of embryos produced was observed relative to the previous year (375,894 in 2015 vs. 391,805 in 2014; $-4.1 \%$ ), similar to what has been observed since 2013, which was probably related to the Brazilian economic recession during this period. This retraction in the activity of the embryo industry ceased the trend of growth in Brazilian numbers observed during the last decade, determined mainly by a significant increase in the use of in vitro embryo production (IVEP) in dairy breeds (Fig. 1).

The increase in dairy IVEP was primarily supported by the commercial availability of sex-sorted semen (Pontes et al., 2010) and caused important changes in the features of the Brazilian embryo market. The most significant one was a shift towards the use of B. taurus breeds for IVEP (Fig. 2). In 2005, zebu beef breeds (mostly Nelore) accounted for $93.8 \%$ of all embryos produced, whereas dairy B. taurus breeds and crossbreds became the most important market for the embryo industry in 2015 , corresponding to $51.8 \%$ of the total number of embryos. The majority of embryos are still transferred fresh, but the percentage of frozenthawed embryos has grown in the past few years, reaching $22.8 \%$ in 2015 , the highest in a decade, likely due to improvements in cryopreservation strategies, such as direct transfer (Sartori et al., 2016). Data from

${ }^{4}$ Corresponding author: henrique.viana@embrapa.br 
2005-2015 also demonstrates that there was no change in the use of in vitro fertilization/culture as the technique of choice for embryo production, both in dairy $(97.2 \%)$ and beef $(90.2 \%)$ breeds.

Table 1. Bovine embryo production in Brazil in 2015, according to genetic group (Bos taurus or Bos indicus), industry (dairy or beef), and technology used (in vivo derived - IVD or in vitro produced - IVP).

\begin{tabular}{lcc}
\hline \multicolumn{1}{c}{ Group } & IVD & IVP \\
\hline B. indicus dairy & 172 & 13,481 \\
B. taurus dairy & 5,759 & 188,853 \\
\hline Subtotal dairy & 5,931 & 202,334 \\
B. indicus beef & 2,042 & 81,636 \\
B. taurus beef & 14,382 & 69,569 \\
\hline Subtotal beef & 16,424 & 151,205 \\
Total & 22,355 & 353,539 \\
\hline
\end{tabular}

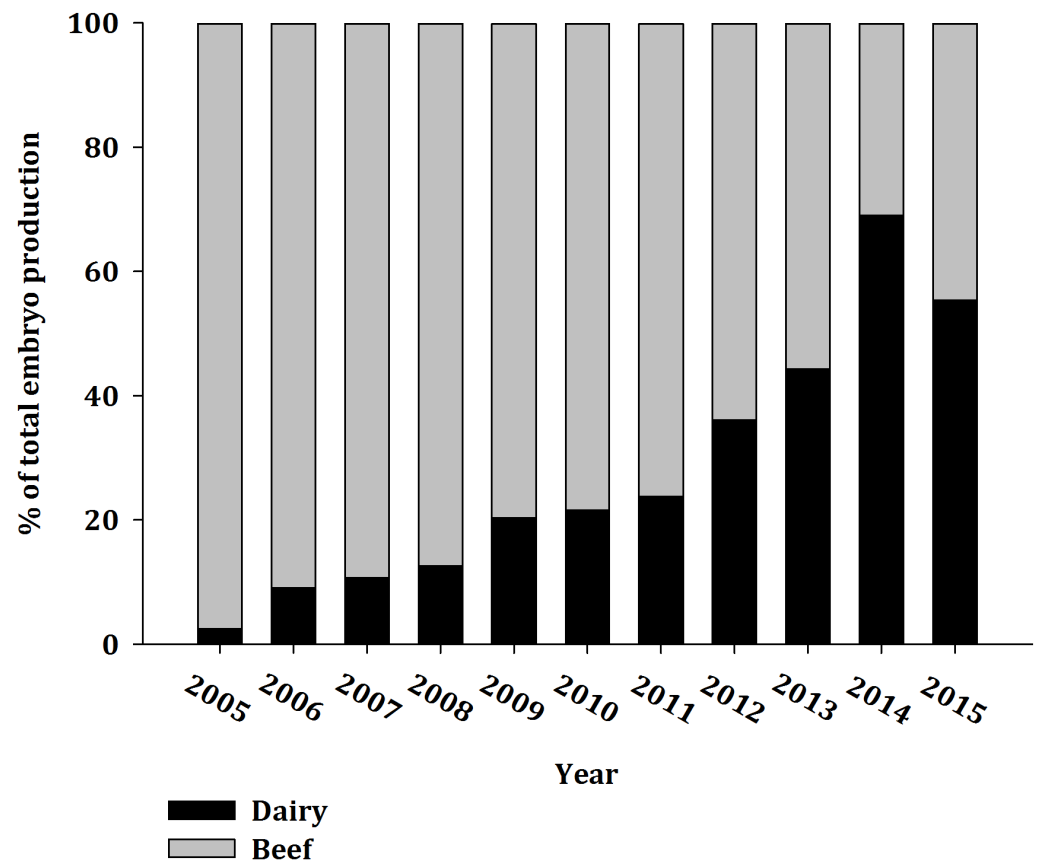

Figure 1. Percentage of Brazilian total embryo production from dairy and beef breeds, from 2005 to 2015.

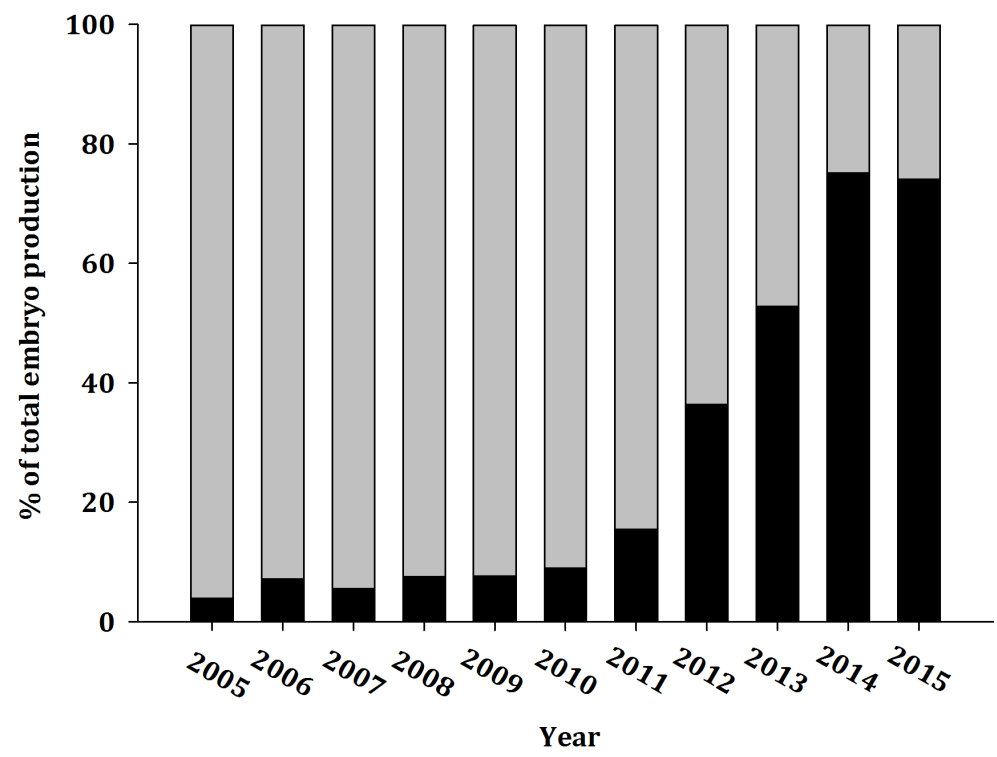

Bos taurus and crossbred $\square$ Bos indicus

Figure 2. Percentage of Brazilian total embryo production from B. taurus and B. indicus breeds, from 2005 to 2015. 


\section{The relevance of embryo technologies}

The success of commercial IVEP initiated an exponential growth in Brazilian embryo production and, since 2010 , the country reports $>300,000$ embryos per year. Despite the scarce number of studies evaluating the economical aspects of IVEP in Brazil, the number of embryos produced undoubtly illustrates the impact of the adoption of in vitro technologies for the embryo industry. Likewise, the growth of IVEP was associated with the parallel development of a chain of suppliers of veterinary services, hormones, media, disposables, equipments, recipients, among others.

Interestingly, if embryo production is analyzed in face of the Brazilian cattle herd size, an apparent paradox is evident. Table 2 shows the evolution of cattle population in the period 1995-2015 and the estimated relative use of artificial isemination (AI) and embryo transfer (ET). These projections demonstrate that the use of embryo technologies increased by a remarkable rate of $726.5 \%$ in the past 20 years; yet, these technologies still reach only $0.33 \%$ of the cows and heifers suitable for reproduction, a very low proportion even considering the low use of other reproductive technologies such as AI (13.3\%). In dairy breeds, the increase in the use of ET was even greater $(2,261.7 \%)$, but the relative use is also very limited $(0.48 \%)$. Therefore, we pose a question: how relevant were embryo technologies for the Brazilian livestock production?

Table 2. Estimated use of artificial insemination (AI) and embryo transfer (ET) as a percentage of the Brazilian cattle herd, 1995-2015.

\begin{tabular}{lccccc}
\hline & 1995 & 2000 & 2005 & 2010 & 2015 \\
\hline Herd size $^{1}$ & $161,227,938$ & $169,875,524$ & $207,156,696$ & $209,541,109$ & $215,199,488$ \\
Heifers and cows $^{2}$ & $42,886,632$ & $45,186,889$ & $55,103,681$ & $55,810,800$ & $57,243,064$ \\
Semen straws sales & $4,180,971$ & $5,769,348$ & $7,028,308$ & $9,637,337$ & $13,700,000$ \\
Use of AI $(\%)^{3}$ & 5.4 & 7.1 & 7.1 & 9.6 & 13.3 \\
Embryos produced & 34,076 & 72,050 & 259,252 & 303,237 & 375,894 \\
Use of ET $(\%)^{4}$ & 0.04 & 0.08 & 0.24 & 0.27 & 0.33 \\
\hline
\end{tabular}

${ }^{1}$ Total number of heads (Instituto Brasileiro de Geografia e Estatística - IBGE, 2016). ${ }^{2}$ Number of females at reproductive age, based on projections by the Brazilian Artificial Insemination Association (ASBIA). ${ }^{3}$ Proportion relative to the number of females at reproductive age. Estimated considering 1.8 straws per successful pregnancy. ${ }^{4}$ Proportion relative to the number of females at reproductive age. Estimated considering 2.0 ET per successful pregnancy in a given recipient.

To address this question, it is important to depict the use of embryo technologies amongst different livestock sectors and production strata. Since the early 2000's, when commercial IVEP began its rise in Brazil, until very recently, most embryos were produced from zebu breeds. As a consequence, these breeds represent the most comprehensive database available. In Table 3 , we estimate the percentage of calves born by ET (conceived by IVP or IVD embryos) relative to all birth records (RGN) reported by the Brazilian Association of Zebu Cattle Breeders (Associação Brasileira dos Criadores de Zebu - ABCZ, 2017) in the period 2005-2015.

Table 3. Estimated percentage of birth records (RGN) from embryo transfers based on records of the Brazilian Zebu Cattle Breeders Association, from 2005 to $2015^{*}$.

\begin{tabular}{lcccccccccccc}
\hline Breed & 2005 & 2006 & 2007 & 2008 & 2009 & 2010 & 2011 & 2012 & 2013 & 2014 & 2015 & Mean \\
\hline Gir & 18.8 & 34.9 & 41.2 & 47.5 & 71.0 & 67.1 & 54.3 & 52.1 & 40.2 & 32.9 & 39.0 & 39.8 \\
Guzerá & 18.9 & 25.3 & 31.1 & 34.7 & 30.9 & 28.5 & 27.1 & 30.3 & 23.5 & 13.7 & 10.8 & 22.7 \\
Indubrasil & 21.8 & 22.8 & 7.8 & 0.5 & 6.8 & 51.1 & 29.0 & 17.8 & 24.0 & 2.3 & 27.8 & 16.3 \\
Nelore & 20.7 & 21.8 & 21.1 & 22.7 & 21.0 & 19.5 & 22.2 & 21.7 & 19.6 & 9.1 & 9.0 & 17.8 \\
Sindi & 0.0 & 0.1 & 13.2 & 19.6 & 29.4 & 26.8 & 21.6 & 28.6 & 21.5 & 25.4 & 28.8 & 16.5 \\
Tababuã & 4.5 & 6.5 & 7.8 & 6.9 & 6.0 & 8.3 & 7.3 & 6.9 & 3.0 & 2.8 & 5.4 & 5.5 \\
Cangaian & 0.0 & 0.0 & 0.0 & 0.0 & 100.0 & 0.0 & 0.0 & 0.0 & 0.0 & 0.0 & 0.0 & 7.7 \\
Brahman & 63.6 & 78.0 & 73.9 & 55.6 & 46.4 & 48.3 & 50.1 & 40.5 & 29.8 & 9.4 & 8.7 & 45.5 \\
Total & 21.5 & 24.1 & 23.7 & 25.1 & 25.0 & 23.7 & 25.1 & 24.0 & 20.3 & 10.2 & 10.1 & 19.7 \\
\hline
\end{tabular}

*Based on a projected $40 \%$ pregnancy rate relative to the total number of embryos transferred in the previous year.

The results of this projection suggest an entirely different scenario compared to the overall ET data, with ET accounting for an impressive $19.7 \%$ of all purebred calves registered by ABCZ. Because zebu breeds are part of most Brazilian beef and dairy herds (either as purebred or crossbreds), any genetic progress in $B$. indicus profoundly affects national livestock productivity indexes. Such impact is likely to have been 
boosted by the intensive use of embryo technologies in the past 10-15 years. Results from the Girolando (Gir x Holstein crosses) breeding program, for example, support this theory. Over 600 of the top 1,000 Girolando cows, ranked according to genetic merit for milk production (Silva et al., 2016), were generated by ET (IVP or IVD embryos).

In Table 3 we provide data to demonstrate that the use of ET was not uniform amongst zebu breeds. In absolute numbers, Nelore accounted for most embryos (76.8\%) produced between 2005 and 2015. Nonetheless, the relative contribution of ET for Nelore calf production $(17.8 \%$ ) was lesser (although more stable) than for some other breeds. On average, ET was used more intensively in Brahman and Gir females (45.5 and $39.8 \%$, respectively), but in both cases these numbers fluctuated, with peaks in 2006 for the former (78.0\%) and 2009 for the latter (71.0\%). In these particular two breeds, such oscillations were probably associated with the transitory use of ET to increase the number of animals rather than for genetic selection, due to the repressed demand for potential oocyte donors.

\section{The Brazilian embryo industry in the world's context}

The remarkable increase in embryo production caused by the use of in vitro technologies also changed Brazil's position in the world rank. In the late 1990's, the Brazilian embryo industry was already very active and the country ranked among the TOP 5 in bovine embryo production (Thibier, 2000). After the rise of commercial IVEP, the participation of Brazil in the world's total numbers increased two-fold (from 14.3\% in 1999 to $33.9 \%$ in 2012) and the country became the largest producer of bovine IVP embryos (Table 4). Interestingly, in the early days of IVEP (up to 2005), the Brazilian embryo market diverged from the trends observed in other countries, particularly from those with relevant embryo production. Therefore, the commercial success of IVEP in Brazil seemed to be a result of particularities of the Brazilian internal market, such as the high economic values and a greater oocyte yield of zebu breeds.

Table 4. Brazilian share in the world's production of bovine embryos in vivo, in vitro, and total, from 2005 to 2015.

\begin{tabular}{|c|c|c|c|c|c|c|c|c|c|}
\hline & \multicolumn{3}{|c|}{ In vivo } & \multicolumn{3}{|c|}{ In vitro } & \multicolumn{3}{|c|}{ Total } \\
\hline & Brazil & World & $\%$ & Brazil & World & $\%$ & Brazil & World & $\%$ \\
\hline 2015 & 22,355 & 660,221 & 3.4 & 353,539 & 612,709 & 57.7 & 375,894 & $1,272,930$ & 29.5 \\
\hline 2014 & 43,337 & 614,464 & 7.1 & 348,468 & 590,359 & 59.0 & 391,805 & $1,204,823$ & 32.5 \\
\hline 2013 & 50,455 & 729,246 & 6.9 & 366,517 & 546,628 & 67.1 & 416,972 & $1,275,874$ & 32.7 \\
\hline 2012 & 52,719 & 699,585 & 7.5 & 334,913 & 443,533 & 75.5 & 387,632 & $1,143,118$ & 33.9 \\
\hline 2011 & 32,646 & 732,862 & 4.5 & 318,116 & 453,471 & 70.2 & 350,762 & $1,186,333$ & 29.6 \\
\hline 2010 & 38,974 & 732,227 & 5.3 & 264,263 & 450,549 & 58.7 & 303,237 & $1,182,776$ & 25.6 \\
\hline 2009 & 42,397 & 704,230 & 6.0 & 255,993 & 378,244 & 67.7 & 298,390 & $1,082,474$ & 27.6 \\
\hline 2008 & 69,527 & 746,250 & 9.3 & 220,425 & 330,953 & 66.6 & 289,952 & $1,077,203$ & 26.9 \\
\hline 2007 & 57,368 & 763,467 & 7.5 & 212,441 & 434,581 & 48.9 & 269,809 & $1,198,048$ & 22.5 \\
\hline 2006 & 83,741 & 777,747 & 10.8 & 204,402 & 441,364 & 46.3 & 288,143 & $1,219,111$ & 23.6 \\
\hline 2005 & 122,210 & 789,972 & 15.5 & 137,042 & 330,647 & 41.4 & 259,252 & $1,120,619$ & 23.1 \\
\hline
\end{tabular}

The recent trends in both Brazilian and international numbers, however, are changing this perception. The use of IVEP has increased consistently in the past 5 years in Europe and North America, considering either absolute numbers $(+71.5$ and $+337.4 \%$; Europe and North America, respectively) or the proportion of total embryo production $(+41.3$ and $+214.5 \%$; respectively), and may possibly replace MOET as the main source of embryos in a near future (Stroud, 2012; Perry, 2013, 2014, 2015, 2016).
Conversely, production of embryos from B. taurus and other dairy breeds is increasing in Brazil, as shown in Fig. 1 and 2. Altogether, the general trend is that features of the embryo industry will become more similar worldwide, in spite of regional differences (Table 5). This will probably contribute to an increase in the demand for international trade of embryos and also stimulates a greater participation of large animal breeding companies in the embryo industry, as we currently see for cattle semen.

Table 5. World production of bovine embryos in 2015, according to region, industry (dairy or beef), and technology used (in vivo derived - IVD or in vitro produced - IVP).

\begin{tabular}{|c|c|c|c|c|c|c|c|c|c|}
\hline \multirow[t]{2}{*}{ Region } & \multicolumn{4}{|c|}{ IVD } & \multicolumn{4}{|c|}{ IVP } & \multirow[t]{2}{*}{ Total } \\
\hline & Dairy & Beef & Subtotal & $\%$ & Dairy & Beef & Subtotal & $\%$ & \\
\hline Africa & 402 & 5,132 & 5,534 & 59.7 & 0 & 3,733 & 3,733 & 40.3 & 9,267 \\
\hline Asia & 16,057 & 89,628 & 105,685 & 91.8 & 1,162 & 8,276 & 9,438 & 8.2 & 115,123 \\
\hline Europe & 104,174 & 23,806 & 127,980 & 90.3 & 12,840 & 940 & 13,780 & 9.7 & 141,760 \\
\hline North America & 127,613 & 232,407 & 360,020 & 62.9 & 105,198 & 106,848 & 212,046 & 37.1 & 572,066 \\
\hline Oceania & 2,178 & 9,009 & 11,187 & 74.2 & 3,892 & 0 & 3,892 & 25.8 & 15,079 \\
\hline South America & 8,636 & 41,179 & 49,815 & 11.9 & 208,752 & 161,068 & 369,820 & 88.1 & 419,635 \\
\hline Total & 259,060 & 401,161 & 660,221 & 51.9 & 331,844 & 280,865 & 612,709 & 48.1 & $1,272,930$ \\
\hline
\end{tabular}

Adapted from Perry (2016). 
The United States of America is currently the global leader in total embryo production (IVD plus IVP; Table 6) and may overtake Brazil's leadership in the production of embryos in vitro, as this technology replaces MOET, i.e., without any further increase in total embryo production. Taking into account the ratio between embryo production and cattle population, the Netherlands is the country that most intensively uses embryo technologies, followed by Canada. According to this criteria, Brazil ranks only 11 th. Nevertheless, considering that Brazil has the largest commercial cattle herd in the world, livestock production is of great importance for the country's economy, and that there is an ongoing pressure to increase cattle productivity, we believe that there is room for a sustainable increase in embryo production in the near future, which may eventually change positions on this ranking.

Table 6. Ranking of countries based on their proportion of embryos produced relative to size of the cattle herd, based on data from the year 2014 .

\begin{tabular}{lcccc}
\multicolumn{1}{c}{ Country } & Embryos produced & Cattle population & Intensity of use of ET (\%) & Rank \\
\hline The Netherlands & 38,637 & $4,169,000$ & 0.93 & $1 \mathrm{st}$ \\
Canada & 87,113 & $12,220,000$ & 0.71 & $2 \mathrm{nd}$ \\
Luxembourg & 1,282 & 198,780 & 0.64 & $3 \mathrm{rd}$ \\
USA & 506,626 & $88,526,000$ & 0.57 & 4 th \\
Finland & 3,617 & 914,439 & 0.40 & 5 th \\
Italy & 19,355 & $5,756,072$ & 0.34 & 6 th \\
Denmark & 4,428 & $1,563,535$ & 0.28 & 7 th \\
Switzerland & 3,397 & $1,562,801$ & 0.22 & 8 th \\
Belgium & 5,138 & $2,477,236$ & 0.21 & 9 th \\
France & 38,422 & $19,095,797$ & 0.20 & 10 th \\
Brazil & 391,805 & $212,366,132$ & 0.18 & 11 th \\
Panama & 2,708 & $1,625,200$ & 0.17 & 12 th \\
Germany & 20,913 & $12,742,190$ & 0.16 & 13 th \\
Hungary & 675 & 782,000 & 0.09 & 0.08 \\
Spain & 4,900 & $6,078,700$ & & 14 th \\
\hline
\end{tabular}

${ }^{\mathrm{T}}$ Food Agriculture Organization (FAO; 2014).

\section{Conclusions}

Predicting the development of the embryo industry is always a complex task, due to the dynamic nature of livestock production and the continuous advance in the 'state of the art' of embryo technologies. Nonetheless, the numbers and trends observed in embryo production in the past decade points to some possible scenarios for the industry in the near future:

1. Despite the current retraction in activity, ET is still used in a low percentage of females and herds in Brazil and, therefore, there is a great potential to increase embryo production as more farms adopt these technologies to increase production and productivity, which was also predicted for AI (Baruselli et al., 2012). A new cycle of expansion in the use of ET, however, is more likely to occur associated to the supply of crossbred calves for dairy and beef farms, instead of animal breeding programs;

2. As first observed in Brazil, IVEP is likely to become the technique of choice for ET worldwide. This will reduce regional differences in embryo production systems, as well as reduce costs and increase the scale of embryo production, probably boosting national and international trading of embryos. Consequently, the international embryo market may become more similar to the semen market;

3. In both scenarios described above, the development of successful cryopreservation strategies is key to further expand the embryo industry, as along with an agreement of sanitary protocols and regulations for the export/import of IVP embryos.

\section{Acknowledgments}

The authors thank the SBTE statistics committee and the Data Retrieval Committee of the IETS for the Brazilian and world embryo production data. They also thank the following breeders associations for the information on embryo transfers: ABCZ, ABCBRH, ABCGPS, ABCG, ABCM, ABC Herd Book Collares, ABCSS.

\section{References}

Associação Brasileira dos Criadores de Zebu (ABCZ). 2017. Estatísticas. Available on: http://www.abcz.org.br/conteudos/tecnica/estatistica_19 39-2016.xls.

Baruselli PS, Sales JNS, Sala RV, Vieira LM, Sa Filho MF. 2012. History evolution and perspectives of timed artificial insemination programs in Brazil. Anim Reprod, 9:139-152.

Food Agriculture Organization (FAO). 2014. Cattle inventory. Available on: http://www.fao.org/ faostat/en/\#data/QA.

Instituto Brasileiro de Geografia e Estatística (IBGE). 2016. Indicadores de produção. Available on: http://www.ibge.gov.br/home/estatistica/indicadores/agr opecuaria. 
Perry G. 2013. 2012 statistics of embryo collection and transfer in domestic farm animals. Embryo Transfer Newsl, 31(4):24-46.

Perry G. 2014. 2013 statistics of embryo collection and transfer in domestic farm animals. Embryo Transfer Newsl, 32(4):14-24.

Perry G. 2015. 2014 statistics of embryo collection and transfer in domestic farm animals. Embryo Transfer Newsl, 33(4):9-18.

Perry G. 2016. 2015 statistics of embryo collection and transfer in domestic farm animals. Embryo Transfer Newsl, 34(4):10-24.

Pontes JH, Silva KC, Basso AC, Rigo AG, Ferreira CR, Santos GM, Sanches BV, Porcionato JP, Vieira PH, Faifer FS, Sterza FA, Schenk JL, Seneda MM. 2010. Large-scale in vitro embryo production and pregnancy rates from Bos taurus, Bos indicus, and indicus-taurus dairy cows using sexed sperm. Theriogenology, 74:1349-1355.

Sartori R, Prata AB, Figueiredo ACS, Sanches BV, Pontes GCS, Viana JHM, Pontes JH, Vasconcelos JLM, Pereira MHC, Dode MAN, Monteiro Jr PLJ, Baruselli PS. 2016. Update and overview on assisted reproductive technologies (ARTs) in Brazil. Anim Reprod, 13:300-312.

Silva MVGB, Martins MF, Cembranelli MAR, Paiva LC, Panetto JCC, Alves BRC, Campos MM, Carvalho BC, Machado MA, Faza DRLR. 2016. Girolando breed genetic improvement program. Sire Summary. Progeny Test Results. June 2016. Juiz de Fora: Embrapa Dairy Cattle. 50 pp. (Embrapa Dairy Cattle. Documents, 190).

Stroud B. 2012. The year 2011 worldwide statistics of embryo transfer in domestic farm animals.. Embryo Transfer Newsl, 30(4):16-26.

Thibier M. 2000. The IETS statistics of embryo transfers in livestock in the world for the year 1999: a new record for bovine in vivo-derived embryos transferred. Embryo Transfer Newsl, 18(4):24-28.

Viana JHM, Siqueira LGB, Palhao MP, Camargo LSA. 2010. Use of in vitro fertilization technique in the last decade and its effect on Brazilian embryo industry and animal production. Acta Sci Vet, 38:s661-s674.

Viana JHM, Siqueira LGB, Palhao MP, Camargo LSA. 2012. Features and perspectives of the Brazilian in vitro embryo industry. Anim Reprod, 9:12-18. 